\title{
Study of quality markers of antiuric acid formula by grey relational analysis
}

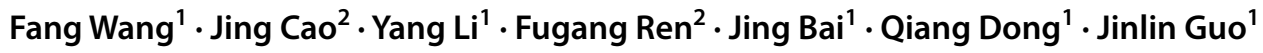

Received: 20 October 2020 / Accepted: 19 January 2021

Published online: 25 May 2021

(c) The Author(s) 2021 OPEN

\begin{abstract}
Gout has become a global problem, antiuric acid formula (AAF) is a clinical prescription highly effective in reducing uric acid levels. In order to find its quality control standards and contribute to the treatment of gout in the future, we adopted high-performance liquid chromatography and orbitrap liquid chromatography-mass spectrometry to establish fingerprints of 13 batches of AAF. The different batches of AAF were tested the activity of inhibit uric acid by the xanthine oxidase inhibition experiment. Grey relational analysis and bio-activity validation to assess the spectrum-effect relationship. Finally, we choose puerarin, calycosin-7-O-beta-D-glucoside and puerarin apioside as the AAF quality control component, and its average content is $6036.006 \mu \mathrm{g} / \mathrm{g}, 296.113 \mu \mathrm{g} / \mathrm{g}$ and $878.285 \mu \mathrm{g} / \mathrm{g}$. As the quality control components of AAF, puerarin, calycosin-7-O-beta-D-glucoside and puerarin apioside can be of great significance for the treatment of gout and gout related research.
\end{abstract}

Keywords Antiuric acid formula · Fingerprint · Pharmacodynamics · Spectrum-effect analysis · Orbitrap LC-MS

\section{Introduction}

Gout and hyperuricemia are common metabolic disorders in humans. Up to 2017, the incidence of gout in China was second only to that of diabetes mellitus, and gout has become the second most widespread metabolic disease $[1,2]$. The prevalence of gout is increasing worldwide, and gout has become a global problem [3]. Drugs such as allopurinol, febuxostat, and probenecid are commonly used to reduce uric acid levels, but their long-term use may cause adverse reactions [4], such as rashes, abnormalities in liver function, allopurinol hypersensitivity, and blood abnormalities, in particular, allopurinol hypersensitivity syndrome. These serious adverse events are rare but can be fatal [5]. The first record of gout in traditional Chinese medicine (TCM) can be traced back to the beginning of the Warring States Period (approximately 403-222 BC) in the Spiritual Pivot of Huangdi's Internal Classic [6]. TCM has been employed in China to alleviate gout, as recorded in the Synopsis of Prescriptions of the Golden Chamber [7, 8] from the Han Dynasty.

Many Chinese medicines could lower uric acid levels [9], including Jiawei Huoxue Simiao decoction, Bixie Chubi decoction, and Xiezhuo Chubi decoction. We found that a formula composed of Puerariae Lobatae Radix, Fructus Mume, Ecklonia kurome, Apium graveolens, and Semen Coicis can significantly reduce the content of uric acid in the body [10]. This formula, which is termed Antiuric Acid Formula (AAF), has anti-inflammatory and analgesic effects, and was proved to be of nontoxic grade in terms of acute

$\square$ Jinlin Guo, guo596@cdutcm.edu.cn| ${ }^{1}$ Key Laboratory of Standardization of Chinese Medicine, Ministry of Education, Key Laboratory of Systematic Research, Development and Utilization of Chinese Medicine Resources in Sichuan Province - Key Laboratory Breeding Base Co-founded by Sichuan Province and MOST, Chengdu University of Traditional Chinese Medicine, No. 1166 Liu-Tai Road, Chengdu 611137, Sichuan, China. ${ }^{2}$ College of Medical Technology, Chengdu University of Traditional Chinese Medicine, Chengdu 611137, Sichuan, China.

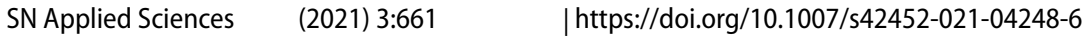


toxicity. For example, the maximum dose in mice reached 1379 times than the adult dose [11]. Previous studies have shown that its active ingredient inhibits xanthine oxidase and reduces the extent of inflammation. This indicates that the formula is a potential antiuric acid drug However, the formula consists of five traditional Chinese medicines, and we did not know which was the effective ingredient or could serve as a quality marker. So the identification of the effective ingredient and a quality marker for quality control became the key to further study.

In the screening of the effective ingredient and quality control markers of traditional Chinese medicines, most researchers have employed TCM fingerprint technology [12]. However, the fingerprints of traditional Chinese medicines cannot explain the correlations between the fingerprints and the efficacy of these medicines. The fingerprints of traditional Chinese medicines were chosen as the basis of research, and the chemical composition and pharmacological action of these medicines were related by analytical methods. A model relationship between the chemical composition and pharmacological action was thus constructed and was termed the spectrum-effect relationship [13]. In recent years, many studies of single traditional Chinese medicines and compound preparations have been carried out with regard to their spectrum-effect relationships [14-16].

Previous research has investigated the pharmacodynamics and safety of AAF by animal experiments [10]. The quality markers of the formula were the most important aspects that remained for follow-up study. We first established the fingerprint of AAF and performed a similarity evaluation. On the basis of a combination of in vitro experiments on the inhibition of xanthine oxidase and grey correlation, the common peaks in high-performance liquid chromatography (HPLC) fingerprints were identified, and the contributions of the corresponding compounds to the inhibition of xanthine oxidase were analyzed. Finally, Orbitrap liquid chromatography-mass spectrometry (LC-MS) was used for qualitative analysis to identify the main index components and to establish standards for the quality control of AAF, which provided a basis for formulating standards for the quality control of other traditional Chinese medicines.

\section{Materials and methods}

\subsection{Reagents and materials}

The AAF clinical prescription was obtained from the Affiliated Hospital of Chengdu University of Traditional Chinese Medicine. It is a medicine and food homology prescription with an extraction rate of $15: 1$ by water extraction and spray drying.

The 13 batches of AAF samples (AAF01-AAF13) were provided by Shanghai Tianyuan Vegetable Products Co., Ltd. (Shanghai, China). Information on these samples is given in Table S16. xanthine, xanthine oxidase, and allopurinol were purchased from Sigma-Aldrich Company (Shanghai, China). The reference materials, namely, puerarin (CAS\# 3681-99-0), ononin (CAS\# 486-62-4), calycosin-7-O-beta-D-glucoside (CAS\# 20633-67-4), puerarin apioside (CAS\# 103654-50-8), berberine (CAS\# 2086-831), palmatine (CAS\# 3486-67-7), glycitin (CAS\# 40246-104), and magnoflorine (CAS\# 2141-09-5), were purchased from Chengdu Pufei De Biotech Co., Ltd. (Sichuan, China). Methanol and acetonitrile were purchased from Fisher Scientific International, Inc. Formic acid was purchased from Chengdu Chron Chemicals Co., Ltd. The ultrapure water used in the experiments was purchased from A.S. Watson Group (Hong Kong) Ltd. All other reagents and chemicals used were analytically pure.

\subsection{Experimental method for inhibition of xanthine oxidase}

The xanthine oxidase inhibition experiments were carried out in the laboratory with reference to methods described in previous research [10]. The uric acid production was calculated according to the increasing absorbance at $290 \mathrm{~nm}$. In 96 well plank, $99 \mu \mathrm{l} \mathrm{pH}=7.40 .02 \mathrm{M}$ PBS buffer, $1 \mu \mathrm{l}$ sample buffer, $50 \mu \mathrm{l} 2 \mathrm{mM}$ fresh xanthine dilute in PBS buffer with little $\mathrm{NaOH}, 50 \mu \mathrm{l} 0.08 \mathrm{U} / \mathrm{mL}$ xanthine oxidase, The mixture was incubated for $30 \mathrm{~min}$ at $37^{\circ} \mathrm{C}$, five biological duplications. The inhibition ratio is expressed in $\%=A_{\text {control }}-A_{\text {sample }} / A_{\text {control }} \times 100 \%$, control is $1 \mu \mathrm{PBS}$ buffer.

\subsection{Preparation of sample solutions and reference standard solution}

Sample powders used in precision weighing experiments were diluted to $0.01 \mathrm{~g} / \mathrm{mL}$ with $80 \%$ methanol( methanol:water $=4: 5 \mathrm{v} / \mathrm{v}), 100 \mathrm{~Hz}$ ultrasonically extracted for $30 \mathrm{~min}$ at $25^{\circ} \mathrm{C}$, centrifuged at $5000 \mathrm{rpm}$ at room temperature for $5 \mathrm{~min}$.

The reference materials, namely, puerarin, ononin, calycosin-7-O-beta-D-glucoside, puerarin apioside, berberine, palmatine, daidzin, and magnoflorine, were diluted to form a $0.2 \mathrm{mg} / \mathrm{mL}$ mother solution with $80 \%$ methanol, further diluted to a concentration of approximately $1 \mu \mathrm{g} / \mathrm{mL}$ with pure water, filtered through a $0.22 \mu \mathrm{m}$ filter membrane. 


\subsection{Instruments and conditions}

The HPLC analysis was carried out using an Agilent 1200 high-performance liquid chromatograph (Agilent Technology, Beijing, China) with a diode array detector (Agilent Technology, Beijing, China) and an Agilent Eclipse $C-18$ column $(250 \times 4.6 \mathrm{~mm}, 5 \mathrm{~m})$. The mobile phase comprised water (A) and methanol (B), the detection wavelength was $290 \mathrm{~nm}$, the column temperature was $25^{\circ} \mathrm{C}$, the flow rate was $0.8 \mathrm{~mL} /$ min, the injection volume was $10 \mu \mathrm{L}$, and the detection time was $60 \mathrm{~min}$. The gradient elution procedure was as follows: 0-15 min: 20\% B; $15-35$ min: 30\% B; 35-50 min: 60\% B; and 50-60 min: $95 \%$ B. The mass spectrometry was carried out by a Thermo Scientific Q Exactive Plus four-stage time-of-flight mass spectrometer, and a Waters ACQUITY UPLC CSH C-18 column $(100 \times 2.1 \mathrm{~mm}, 1.7 \mathrm{~m})$ was used. The mobile phase comprised acetonitrile (A) and water (B) and contained $0.1 \%$ formic acid. The gradient elution sequence was as follows: 0-2 min: 5\% A; 2-47 min: 95\% A and 47-50 min: 5\% A. The injection volume was $5 \mu \mathrm{L}$, the flow rate was $0.3 \mathrm{~mL} /$ min, and the column temperature was $40^{\circ} \mathrm{C}$. In the HPLC verification process, the contents of the reference compounds were determined by an Agilent Eclipse $\mathrm{C}-18$ column $(250 \times 4.6 \mathrm{~mm}, 5 \mathrm{~m})$. The elution gradient, flow rate, and column temperature were the same as those used in the above HPLC analysis.

\subsection{Similarity analysis}

The fingerprints of the 13 batches of AAF were compared and analyzed by the system for the similarity evaluation of chromatographic fingerprints of traditional Chinese medicines (version 2012). When matching results were obtained, a control map of the HPLC fingerprints was generated by software calculations, and the common peaks were calibrated.

\subsection{Grey relational analysis}

According to the preliminary method devised by the research group for analyzing sequences [10], the correlation coefficients and degrees of correlation are calculated, and the degrees of correlation are ranked from the highest to the lowest. The correlation order is thus obtained, which can directly represent the degrees of correlation between subsequences and the parent sequence. The greater is the degree of correlation, the closer is the relationship between the subsequence and the parent sequence. On this basis, relationships between common characteristic peaks in HPLC fingerprints and the related clinical efficacy can be established.

\section{Results and discussion}

\subsection{Fingerprints of AAF}

The conditions employed to obtain the HPLC fingerprints and the methodology adopted in obtaining the test results [10] are shown in Figures S1-S6. The HPLC fingerprints and control fingerprints of 13 batches of AAF were obtained by similarity analysis and shown in Fig. 1. The results of the similarity matching are shown in Tables S7. It can be seen that the similarity between the different batches of AAF was high, and the similarity values were all greater than 0.8 . Ten similar peaks were identified from the similarity analysis. The results of the statistical analysis of retention times and peak areas are shown in Tables S8 and S9.

\subsection{Results for the spectrum effect of AAF}

\subsubsection{Results of xanthine oxidase inhibition tests}

The prototypical xanthine oxidase inhibitor allopurinol has been the cornerstone of the clinical management of gout and conditions associated with hyperuricemia for several decades [17]. AAF can inhibit the activity of xanthine oxidase, althoungh the effects not good as allopurinol, as shown in Table 1.

\subsubsection{Results of analysis of grey degree of association}

Grey correlation analysis, which has been widely used in various fields, is an effective means of studying fuzzy systems [18-20]. When grey correlation analysis is carried out, transformation of the mean values is first performed to make the dimensions consistent. In particular, the average value for each sequence needs to be obtained, and then the original data for each corresponding sequence are divided by the average value. The resulting quotients constitute the mean sequence, as listed in Table S10.

The absolute differences between the rates of inhibition of xanthine oxidase and the results of normalization of the relative areas of the ten common characteristic peaks in the HPLC fingerprints are shown in Table 2.

According to the formula used for calculating the correlation coefficient, when the resolution coefficient $\rho$ is 0.5 the optimal result is obtained, and hence we used $\rho=0.5$ in this experiment. The correlation coefficients for the relationships between the ten common characteristic peaks and the rates of inhibition of xanthine oxidase were obtained by this formula, as shown in Table 3. The degrees of correlation were calculated and the results were sorted, as shown in Table 4. The ten common characteristic peaks exhibited high degrees of correlation with the rates of 

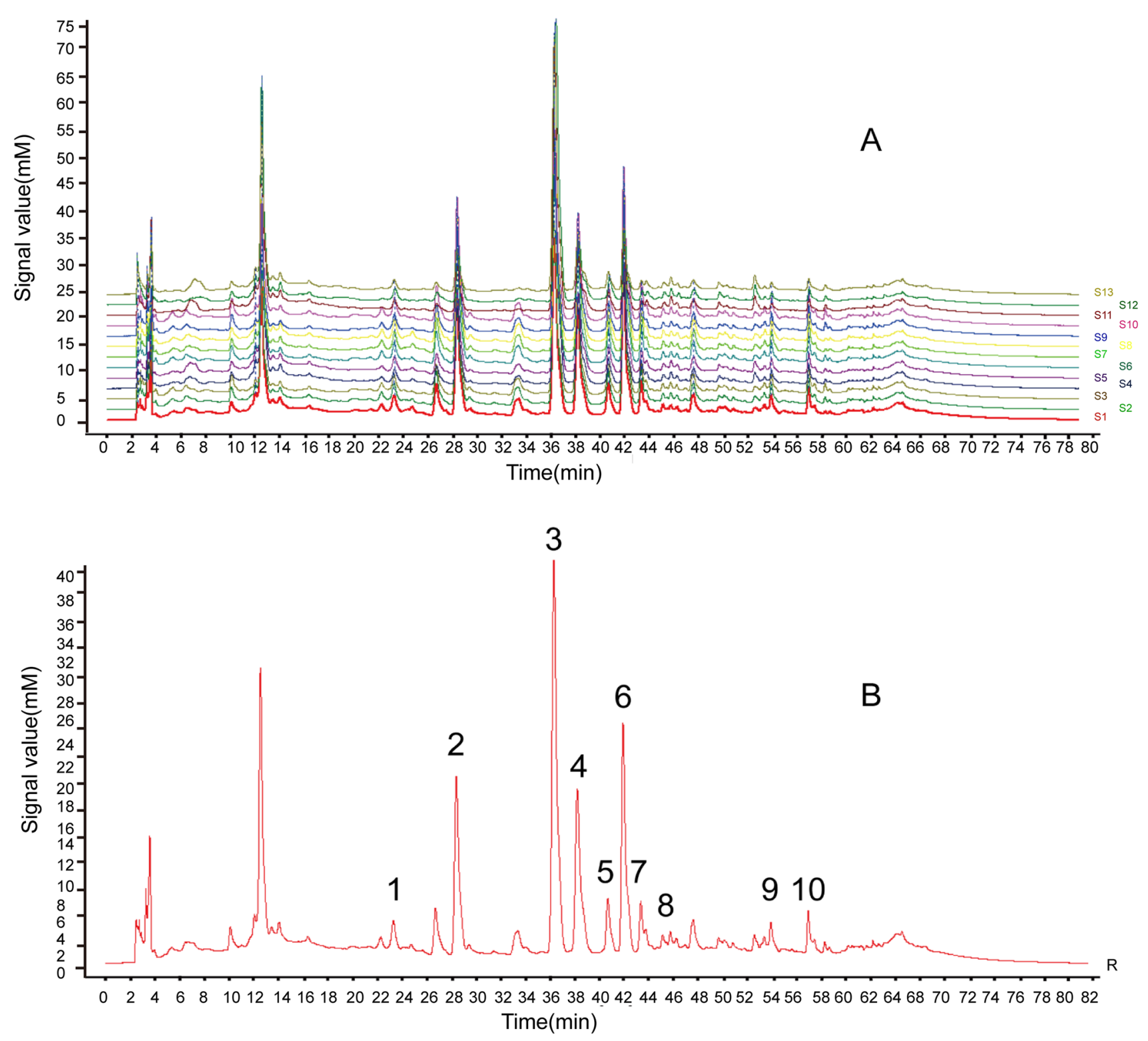

Fig. 1 High-performance liquid chromatography (HPLC) fingerprints of Antiuric Acid Formula (AAF). a HPLC fingerprints of AAF batches (S1S13: AAF01-AAF13); b Comparative HPLC fingerprint of AAF (1-10: common peaks)

inhibition of xanthine oxidase. The contributions of the compounds corresponding to the ten common peaks to the inhibition of xanthine oxidase were investigated as follows: peak $10>$ peak $9>$ peak $1>$ peak $8>$ peak $7>$ peak $5>$ peak $2>$ peak $4>$ peak $6>$ peak 3 . The degrees of correlation between peak 10 , peak 9 , peak 1 , peak 8 , peak 7 , peak 5 , and the effect of AAF were all greater than 0.85 . This shows that the ingredients corresponding to these six peaks are highly correlated with the pharmacodynamic effect of AAF.

\subsection{Identification of index components and establishment of quality standards}

The data were processed through TraceFinder 3.3 software [21]. The settings used for the screening parameters are shown in Table S11. The result of the software matching is then manually screened to filter the data of "all fragment ions match", and peaks for which the retention time was too short were excluded (retention time was $<1 \mathrm{~min}$, insufficient resolution on the column). According to the results of the software calculations, the parent and fragment ions of each inferred substance were located on the total ion chromatogram, and the main fragment ions 
Table 1 Rates of inhibition of xanthine oxidase by samples with different concentrations (mean \pm standard deviation) $(n=5)$

Table 2 Absolute differences between xanthine oxidase inhibition rates and normalized peak areas
Table 3 Correlation coefficients for relationships between characteristic peaks and xanthine oxidase inhibition rates

\begin{tabular}{|c|c|c|c|c|c|c|}
\hline \multirow[t]{2}{*}{ Sample } & \multicolumn{6}{|c|}{ Inhibition rate $(\%)$ at respective concentration $(\mu \mathrm{g} / \mathrm{mL})$} \\
\hline & 5 & 10 & 15 & 25 & 50 & 100 \\
\hline Allopurinol & $5.50 \pm 0.93$ & $6.33 \pm 0.81$ & $8.65 \pm 1.13$ & $10.43 \pm 0.77$ & $13.85 \pm 2.01$ & $23.97 \pm 2.95$ \\
\hline AAF01 & $4.51 \pm 0.69$ & $5.45 \pm 0.71$ & $7.54 \pm 0.78$ & $8.38 \pm 2.20$ & $10.30 \pm 0.28$ & $11.55 \pm 0.48$ \\
\hline AAF02 & $3.83 \pm 1.13$ & $4.79 \pm 1.81$ & $6.36 \pm 2.75$ & $8.36 \pm 1.52$ & $10.41 \pm 1.60$ & $11.98 \pm 1.15$ \\
\hline AAF03 & $3.60 \pm 0.54$ & $5.19 \pm 1.75$ & $6.19 \pm 0.75$ & $6.78 \pm 1.04$ & $8.79 \pm 1.66$ & $9.87 \pm 2.06$ \\
\hline AAF04 & $3.06 \pm 1.36$ & $4.47 \pm 0.97$ & $5.47 \pm 0.15$ & $7.19 \pm 0.33$ & $7.91 \pm 0.77$ & $10.02 \pm 1.36$ \\
\hline AAF05 & $4.20 \pm 0.51$ & $5.15 \pm 0.68$ & $6.78 \pm 0.89$ & $8.35 \pm 1.21$ & $9.25 \pm 1.94$ & $10.28 \pm 2.66$ \\
\hline AAF06 & $3.67 \pm 0.90$ & $4.59 \pm 0.89$ & $5.01 \pm 1.27$ & $6.18 \pm 1.53$ & $7.02 \pm 0.33$ & $9.52 \pm 1.65$ \\
\hline AAF07 & $4.18 \pm 0.54$ & $5.61 \pm 0.86$ & $7.28 \pm 1.03$ & $8.03 \pm 0.44$ & $12.22 \pm 1.54$ & $14.31 \pm 2.48$ \\
\hline AAF08 & $4.07 \pm 1.44$ & $5.95 \pm 1.64$ & $6.96 \pm 1.42$ & $9.48 \pm 0.89$ & $10.91 \pm 0.65$ & $13.23 \pm 0.40$ \\
\hline AAF09 & $4.02 \pm 0.42$ & $5.32 \pm 0.89$ & $6.50 \pm 0.65$ & $7.56 \pm 1.42$ & $8.53 \pm 1.63$ & $10.52 \pm 1.44$ \\
\hline AAF10 & $3.97 \pm 0.54$ & $5.35 \pm 0.53$ & $6.54 \pm 1.03$ & $7.94 \pm 1.01$ & $9.80 \pm 0.16$ & $11.46 \pm 3.15$ \\
\hline AAF11 & $4.20 \pm 0.65$ & $6.56 \pm 1.36$ & $7.27 \pm 1.72$ & $8.67 \pm 0.86$ & $9.89 \pm 1.82$ & $10.70 \pm 2.76$ \\
\hline AAF12 & $4.49 \pm 0.33$ & $5.01 \pm 0.82$ & $6.15 \pm 1.51$ & $7.27 \pm 1.80$ & $8.67 \pm 1.33$ & $11.88 \pm 1.91$ \\
\hline AAF13 & $3.88 \pm 0.66$ & $5.80 \pm 1.34$ & $6.56 \pm 0.89$ & $7.90 \pm 0.78$ & $8.32 \pm 1.17$ & $10.45 \pm 2.07$ \\
\hline
\end{tabular}

\begin{tabular}{|c|c|c|c|c|c|c|c|c|c|c|}
\hline \multirow[t]{2}{*}{ Batch number } & \multicolumn{10}{|c|}{ Peak number } \\
\hline & 1 & 2 & 3 & 4 & 5 & 6 & 7 & 8 & 9 & 10 \\
\hline AAF01 & 0.100 & 1.569 & 2.802 & 1.541 & 0.299 & 1.886 & 0.312 & 0.157 & 0.101 & 0.078 \\
\hline AAF02 & 0.106 & 1.552 & 2.751 & 1.540 & 0.292 & 1.867 & 0.306 & 0.150 & 0.096 & 0.141 \\
\hline AAF03 & 0.117 & 1.575 & 2.796 & 1.600 & 0.310 & 1.917 & 0.333 & 0.166 & 0.092 & 0.106 \\
\hline AAF04 & 0.109 & 1.595 & 2.781 & 1.558 & 0.305 & 1.887 & 0.324 & 0.163 & 0.115 & 0.162 \\
\hline AAF05 & 0.086 & 1.205 & 4.191 & 1.380 & 0.273 & 1.531 & 0.125 & 0.118 & 0.006 & 0.056 \\
\hline AAF06 & 0.100 & 1.229 & 4.175 & 1.392 & 0.277 & 1.550 & 0.134 & 0.124 & 0.010 & 0.058 \\
\hline AAF07 & 0.068 & 1.509 & 2.776 & 1.537 & 0.267 & 1.858 & 0.282 & 0.126 & 0.074 & 0.071 \\
\hline AAF08 & 0.072 & 1.533 & 2.780 & 1.532 & 0.275 & 1.862 & 0.291 & 0.134 & 0.084 & 0.114 \\
\hline AAF09 & 0.093 & 1.569 & 2.864 & 1.597 & 0.305 & 1.924 & 0.323 & 0.163 & 0.108 & 0.001 \\
\hline AAF10 & 0.086 & 1.542 & 2.797 & 1.555 & 0.292 & 1.878 & 0.309 & 0.151 & 0.112 & 0.131 \\
\hline AAF11 & 0.061 & 0.756 & 5.537 & 1.112 & 0.249 & 1.057 & 0.006 & 0.053 & 0.086 & 0.013 \\
\hline AAF12 & 0.031 & 0.761 & 5.548 & 1.109 & 0.200 & 1.064 & 0.012 & 0.041 & 0.071 & 0.002 \\
\hline AAF13 & 0.038 & 0.762 & 5.593 & 1.097 & 0.209 & 1.082 & 0.007 & 0.053 & 0.092 & 0.023 \\
\hline
\end{tabular}

\begin{tabular}{lllllllllll}
\hline Batch number & \multicolumn{2}{l}{ Peak number } \\
\cline { 2 - 11 } & 1 & 2 & 3 & 4 & 5 & 6 & 7 & 8 & 9 & 10 \\
\hline AAF01 & 0.986 & 0.498 & 0.352 & 0.503 & 0.870 & 0.450 & 0.864 & 0.950 & 0.985 & 1.000 \\
AAF02 & 0.993 & 0.503 & 0.357 & 0.505 & 0.882 & 0.454 & 0.875 & 0.965 & 1.000 & 0.971 \\
AAF03 & 0.984 & 0.501 & 0.355 & 0.497 & 0.872 & 0.449 & 0.860 & 0.953 & 1.000 & 0.990 \\
AAF04 & 1.000 & 0.502 & 0.359 & 0.509 & 0.884 & 0.457 & 0.875 & 0.965 & 0.996 & 0.966 \\
AAF05 & 0.964 & 0.637 & 0.334 & 0.605 & 0.887 & 0.580 & 0.946 & 0.950 & 1.000 & 0.977 \\
AAF06 & 0.959 & 0.632 & 0.335 & 0.603 & 0.887 & 0.577 & 0.944 & 0.948 & 1.000 & 0.978 \\
AAF07 & 1.000 & 0.503 & 0.350 & 0.498 & 0.880 & 0.449 & 0.872 & 0.961 & 0.996 & 0.998 \\
AAF08 & 1.000 & 0.500 & 0.351 & 0.500 & 0.878 & 0.450 & 0.870 & 0.960 & 0.992 & 0.972 \\
AAF09 & 0.939 & 0.477 & 0.333 & 0.473 & 0.825 & 0.427 & 0.816 & 0.898 & 0.930 & 1.000 \\
AAF10 & 1.000 & 0.505 & 0.354 & 0.503 & 0.878 & 0.453 & 0.869 & 0.958 & 0.983 & 0.971 \\
AAF11 & 0.981 & 0.787 & 0.334 & 0.715 & 0.919 & 0.725 & 1.000 & 0.983 & 0.972 & 0.997 \\
AAF12 & 0.989 & 0.785 & 0.334 & 0.715 & 0.933 & 0.723 & 0.996 & 0.986 & 0.976 & 1.000 \\
AAF13 & 0.989 & 0.788 & 0.334 & 0.720 & 0.933 & 0.723 & 1.000 & 0.984 & 0.971 & 0.994 \\
\hline
\end{tabular}


Table 4 Order of degrees of correlation between characteristic peaks and xanthine oxidase inhibition rates

\begin{tabular}{lll}
\hline Correlation ranking & Peak number & $\begin{array}{l}\text { Degree of } \\
\text { correlation }\end{array}$ \\
\hline 1 & 10 & 0.986 \\
2 & 9 & 0.985 \\
3 & 1 & 0.983 \\
4 & 8 & 0.959 \\
5 & 7 & 0.907 \\
6 & 5 & 0.887 \\
7 & 2 & 0.586 \\
8 & 4 & 0.565 \\
9 & 6 & 0.532 \\
10 & 3 & 0.345 \\
\hline
\end{tabular}

generated from the parent ion were identified. Twelve possible components with moderate retention times were found. Reference materials were analyzed under the same chromatographic and mass spectrometric conditions, and the retention times corresponding to the chromatographic peaks and the mass/charge ratios $(\mathrm{m} / \mathrm{z})$ of the parent and fragment ions were compared. Each reference standard was matched with a component corresponding to a peak in the fingerprint using the corresponding results of analysis by HPLC. The substances that were successfully compared were analyzed by HPLC together with the corresponding reference substances, and the respective fingerprints were matched. For each successfully matched substance, the standard curve was drawn, its content was determined, and a quality control standard was established.

Orbitrap LC-MS analysis can be carried out in positive or negative ion mode. In negative ion mode, most of the peaks are the same as in positive ion mode, but the number of peaks is smaller, the peak shape is worse, and the peak intensity is lower, as shown in Fig. S7. Hence, positive ion mode was used to analyze the index components.

\subsubsection{Qualitative analysis of the primary mass spectroscopic peaks}

Under the set experimental conditions, each sample was analyzed to obtain its total ion chromatogram, as shown in Fig. 2. The $m / z$ values of the parent and fragment ions were completely matched with those of the corresponding parent and fragment ions recorded in the OTCML database. The first 12 components were selected according to the intensity of the corresponding peak in decreasing order: see Table S12. These 12 components were initially chosen for verification.

\subsubsection{Verification of major mass spectrometric peaks}

Nine reference solutions were analyzed to verify the main mass spectrometric peaks, as shown in Table S13 and Fig. S8. Puerarin, berberine, palmatine, daidzin, magnoflorine, calycosin-7-O-beta- $d$-glucoside, ononin, and puerarin apioside all coincided with the sample in terms of retention time and $m / z$ values of the parent and fragment ions. However, the retention time of vitexin in the sample was inconsistent with that of the reference, which excluded the possibility that component 5 was vitexin. According to the OTCML database and $\mathrm{m} / \mathrm{z}$ values of fragment ions listed in the literature, as shown in Table S15, it was suggested that component 5 should be an isomer of vitexin, which was suspected to be genistein-8-C-glucoside [22].

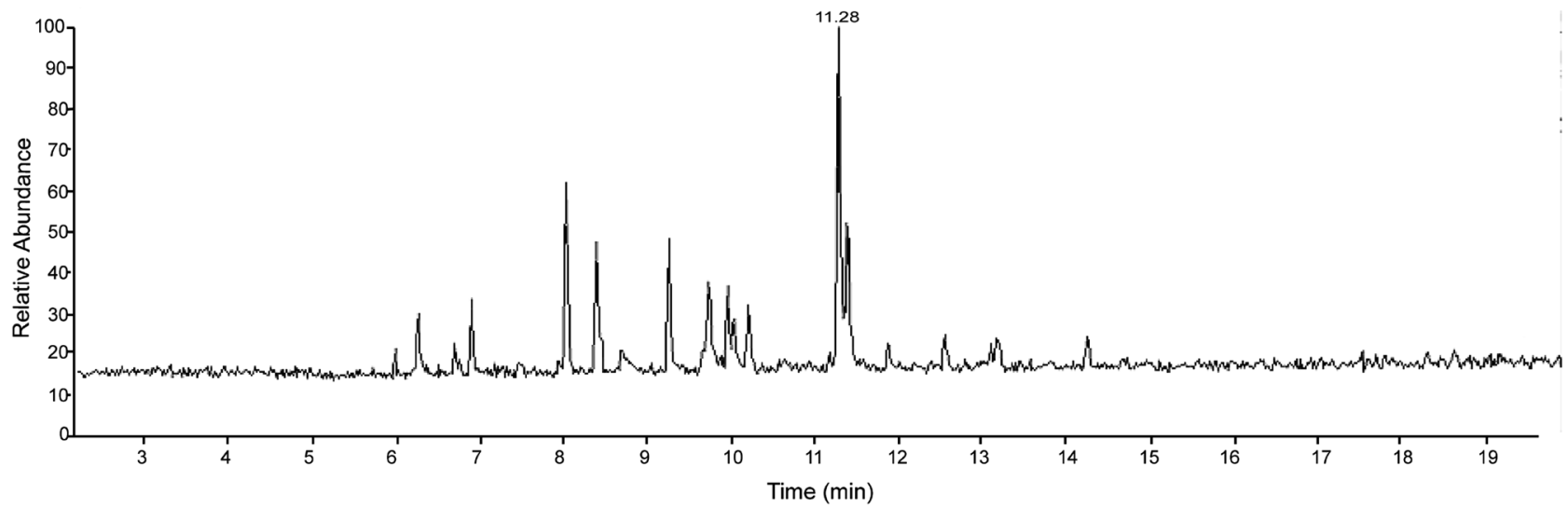

Fig. 2 Total ion chromatogram of the sample solution 


\subsubsection{HPLC verification of eight components}

The remaining reference materials, namely, puerarin, berberine, palmatine, daidzin, magnoflorine, calycosin-7-0-beta- $d$-glucoside, ononin, and puerarin apioside, were verified by HPLC. As a result, only puerarin, ononin, calycosin-7-O-beta-d-glucoside, and puerarin apioside exhibited strong UV absorption under the verification conditions, and their control fingerprints could match the sample fingerprint. These compounds corresponded to peak 3 , peak 9 , peak 8 , and peak 7 , respectively, as shown by Fig. 3.

\subsubsection{Determination of four identified components}

HPLC analysis of solutions of the four remaining reference compounds was performed with different concentration gradients. Standard curves with peak area as the longitudinal coordinate and concentration as the transverse coordinate were obtained, and the corresponding linear regression equations were deduced, as shown in Fig. S9 and Table 5.

The contents of the four verified ingredients in the test sample are shown in Table S14. The content of puerarin in AAF was 4319.118-8324.784 $\mu \mathrm{g} / \mathrm{g}$, with an average value of $6036.006 \mu \mathrm{g} / \mathrm{g}$. The content of puerarin apioside was $535.988-1786.615 \mu \mathrm{g} / \mathrm{g}$, with an average value of $878.285 \mu \mathrm{g} / \mathrm{g}$. The ononin content was $77.946-353.845 \mu \mathrm{g} / \mathrm{g}$, with an average value of $232.944 \mu \mathrm{g} / \mathrm{g}$. Finally, the calycosin-7-O-beta- $d$-glucoside content was $213.181-345.337 \mu \mathrm{g} / \mathrm{g}$, with an average value of $296.113 \mu \mathrm{g} / \mathrm{g}$.

The results showed that the data for puerarin, ononin, calycosin-7-O-beta-D-glucoside, and puerarin apioside matched the fingerprint of the sample. The other four reference materials may not exhibit UV absorption and cannot give rise to chromatographic peaks under the conditions used for verification, and hence they cannot produce control fingerprints that could match the fingerprint of the sample. However, the differences between the contents of the four main index components in different batches of AAF were large, which may have been caused by differences in the sources of the original drugs and differences in the process and yield in production. Among these four components, the difference in the content of ononin was the largest. In view of this, ononin is not recommended as a quality control standard.

\subsubsection{Experimental validation of three bio-active components}

To further investigate the inhibition of xanthine oxidase on three interested compounds, allopurinol as positive control, stabler AAF01 as total component reference. To verify the inhibition of xanthine oxidase of low, medium and high proportion gradients of puerarin, puerarin apigenin, calycosin-7-O-beta-D-glucoside, the results are shown in Table 6. The three components has xanthine oxidase inhibitory effect, and has a certain concentration dependence, although the effect is not as good as AAF compound or positive drugs.

\section{Conclusions}

AAF is widely used in clinical treatment of hyperuricemia. In previous, we has investiated its related efficacy and acute toxicity through animal experiments in vivo [11], confirmed AAF is safe and non-toxic in the range of clinical dosage, and has obvious antiuric acid, antiinflammatory and analgesic effect. The similarity value of 13 batches of samples could reach more than 0.8 . However, the common peak area of different batches of AAF is quite different, so the quality cannot be controlled by fingerprint alone, it is necessary to analyze the pharmaceutical effect.

Considering the animal experiments in vivo are timeconsuming and expensive, we use the inhibition rate of AAF on xanthine oxidase as the source of pharmacological data. The contribution of the fingerprin common peak to the inhibition of xanthine oxidase was obtained by grey correlation degree method. Spectral effect analysis shows the contribution the order of the four main components to reducing uric acid ranges was as follows: Ononin > Puerarin apioside $>$ Calycosin-7-O-beta-D-glucoside $>$ Puerarin. However, the content of Ononin in different batches of compound is quite different, it was not recommended as a quality control index. Therefore, the quality control standard of AAF was established by using Puerarin, Calycosin-7-O-beta-D-glucoside and Puerarin apioside.

It has been reported that puerarin reduces uric acid production by inhibiting xanthine oxidase activity [23], and promote uric acid excretion in tubular epithelial cells [24]. The use of puerarin as anti-hyperuricemia agent is consistent with previous studies [25]. Studies have shown that calycosin-7-O-beta-D-glucoside alleviates antioxidant stress and protects the integrity of the myocardium, neurons, and blood-brain barrier [26-28], but its role in uric acid or hyperuricemia is rarely reported. It is also the first report that puerarin apigenin has the effect of reducing uric acid, and specific mechanisms need to be followed up.

Some quality control methods, like Ultrahigh-performance liquid chromatography have been widely applicated for compound drugs, however index components identified by these methods reflect the compositions of 

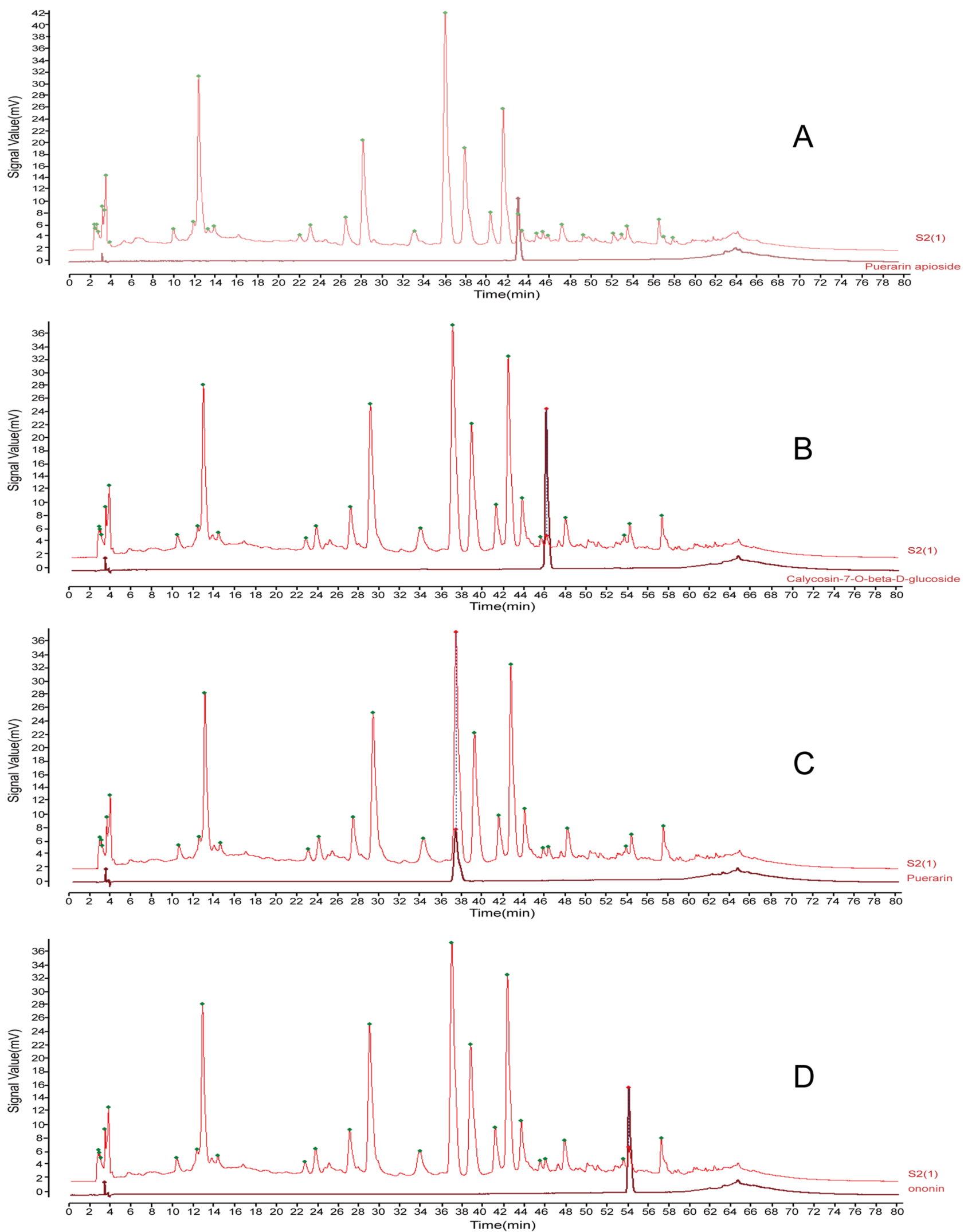

Fig. 3 HPLC chromatograms of a puerarin apioside; $\mathbf{b}$ calycosin-7-O-beta-D-glucoside; $\mathbf{c}$ puerarin, $\mathbf{d}$ ononin 
Table 5 Data for linear regression of standard curves for reference compounds

\begin{tabular}{lll}
\hline Material & Regression equation & $\mathrm{R}^{2}$ \\
\hline Puerarin & $\mathrm{y}=1202 \mathrm{x}+17.22$ & 0.9997 \\
Puerarin apioside & $\mathrm{y}=993.49 \mathrm{x}+37.24$ & 0.9989 \\
$\begin{array}{l}\text { Ononin } \\
\begin{array}{l}\text { Calycosin-7-O-beta-D-glu- } \\
\text { coside }\end{array}\end{array}$ & $\mathrm{y}=1403.2 \mathrm{x}+3.44$ & 1 \\
\hline
\end{tabular}

Table 6 The xanthine oxidase inhibition activities of interested compounds

\begin{tabular}{llr}
\hline Sample & $\begin{array}{l}\text { Concentration }(\mu \mathrm{g} / \\
\mathrm{mL})\end{array}$ & Inhibition rate $(\%)$ \\
\hline Allopurinol & 1 & $8.55 \pm 0.38$ \\
& 10 & $11.99 \pm 1.52$ \\
AAF01 & 100 & $43.48 \pm 1.21$ \\
& 1 & $6.98 \pm 1.80$ \\
& 10 & $9.59 \pm 1.71$ \\
Puerarin & 100 & $21.05 \pm 1.14$ \\
& 4 & $7.75 \pm 1.47$ \\
Puerarin apigenin & 60 & $12.67 \pm 0.58$ \\
& 800 & $18.21 \pm 0.67$ \\
Calycosin-7-O-beta- & 0.5 & $6.63 \pm 1.54$ \\
D-glucoside & 10 & $11.75 \pm 0.64$ \\
& 150 & $17.05 \pm 0.89$ \\
& 0.2 & $8.15 \pm 0.55$ \\
& 2.5 & $14.67 \pm 1.41$ \\
& 30 & $18.13 \pm 0.13$
\end{tabular}

Concentration gradients for the three compounds are set by value rages of proportion in the AAF compound, as shown in Table S14

medicinal materials or compounds and cannot be correlated with the efficacy of drugs. Grey correlation analysis does not require too much sample size, which is suitable for real-time and rapid analysis. It is an effective means to study fuzzy systems such as traditional Chinese medicine and compound. Although the nonlinear relationship between factors is easy to produce wrong analysis and judgment, its evaluation method is simple to calculate, the information reflected in the evaluation process is comprehensive and complete, the evaluation results are accurate and effective, and can reflect the advantages and disadvantages of different grade samples. It is a simple and effective evaluation method.

In present study, a combination of fingerprints and grey correlation analysis was employed to identify the components that contributed to the fingerprints and the active ingredients that inhibited xanthine oxidase. This method will provide new ideas for the study of quality markers in TCM, and contribute to the study of gout.

\section{Supplementary materials}

The following are available online at www.mdpi.com/xxx/ s1, Fig. S1: Comparison of Different Extraction Solvents, Fig. S2 Comparison of different extraction methods, Fig. S3 Comparison of different mobile phases, Fig. S4 Comparison of different flow rate, Fig. S5 Comparison of different wave length, Fig. S6 Methodology investigation, Fig. S7 Pattern of positive and negative ions in samples, Fig. S8 Mass spectra of 9 standard products, Fig. S9 linear regression curve, Table S1 Retention time of stability test $(n=5)$, Table S2 Peak area of stability test $(n=5)$, Table S3 Retention time of precision test $(n=5)$, Table S4 Peak area of precision test $(n=5)$, Table S5 Retention time of repeatability test $(n=5)$, Table S6 Peak area of repeatability test $(n=5)$, Table S7 Similarity Matching Results of the HPLC Fingerprint of the AAF, Table $S 8$ Retention time of mutual peaks, Table S9 Peak area of mutual peaks, Table S10Normalized results of relative peak area, Table S11 Setting of screening parameters, Table S12 Parameters of LC-MS, Table S13 9 Parameters of LC-MS, Table S14 Result of content determination, Table S15 Parameters of LC-MS in references, Table S16 Sample Information.

Acknowledgements Shanghai Tianyuan Vegetable Products Co., Ltd. Provided the 13 batches of samples (AAF01 13).

Author contributions JG conceived this study, designed the experimental plan. FW performed data analysis, writing-original draft. JC participated in data analysis. YL and FR revised and reviewed the manuscript. JB and QD participated in sample preparing. All authors read and approved the final manuscript.

Funding This research was funded by the National Natural Science Foundation of China $(81872959,81373920,30801522)$, Sichuan Youth Science and Technology Foundation (2019CXTD0055), China Scholarship Council (201708150027).

\section{Compliance with ethical standards}

Conflict of interest The authors declare that they have no conflicts of interest.

Open Access This article is licensed under a Creative Commons Attribution 4.0 International License, which permits use, sharing, adaptation, distribution and reproduction in any medium or format, as long as you give appropriate credit to the original author(s) and the source, provide a link to the Creative Commons licence, and indicate if changes were made. The images or other third party material in this article are included in the article's Creative Commons licence, unless indicated otherwise in a credit line to the material. If material is not included in the article's Creative Commons licence and your intended use is not permitted by statutory regulation or exceeds the permitted use, you will need to obtain permission directly from the copyright holder. To view a copy of this licence, visit http://creativecommons. org/licenses/by/4.0/. 


\section{References}

1. Lixin G (2014) Discussion on the treatment of hyperuricemia from "Chinese Expert Consensus for Hyperuricemia and Gout Treatment." Drug Eval 11(01):21-31

2. Choi BG, Kim DJ, Baek MJ, Ryu YG, Kim SW, Lee MW, Park JY, Noh YK, Choi SY, Byun JK, Shim MS, Mashaly A, Li H, Park Y, Jang WY, Kim W, Kang JH, Choi JY, Park EJ, Park SH, Lee S, Na JO, Choi CU, Kim EJ, Park CG, Seo HS, Oh DJ, Rha SW (2018) Hyperuricaemia and development of type 2 diabetes mellitus in Asian population. Clin Exp Pharmacol Physiol 45(6):499-506. https://doi.org/ 10.1111/1440-1681.12911

3. Kuo CF, Grainge MJ, Zhang W, Doherty M (2015) Global epidemiology of gout: prevalence, incidence and risk factors. Nat Rev Rheumatol 11(11):649-662. https://doi.org/10.1038/nrrheum. 2015.91

4. Ito K, Ueda Y, Miyazawa H, Kaku Y, Hirai K, Hoshino T, Nabata A, Mori H, Yoshida I, Ookawara S, Tabei K (2014) Acute severe liver dysfunction induced by febuxostat in a patient undergoing hemodialysis. CEN Case Rep 3(2):158-161. https://doi.org/10. 1007/s13730-014-0109-2

5. Stamp L, Dalbeth N (2017) Urate-lowering therapy for asymptomatic hyperuricaemia: a need for caution. Semin Arthritis Rheum 46(4):457-464. https://doi.org/10.1016/j.semarthrit. 2016.07.015

6. Hongbin Z (2009) Detailed explanation of Huangdi's Internal Classic in vernacular. Liaoning Sci Tech Publi House, Shenyang, pp 266-275p

7. Zhuxing W (2011) Detailed explanation of Huangdi's Internal Classic in vernacular. Tianjin Sci Tech Press, Tianjin, pp 387-388p

8. Xinxing L, Guisheng L (2004) Gout. Chongqing Univ Press, Chongqing, $\mathrm{p} 108 \mathrm{p}$

9. Kunhao L, Shiwei Y (2018) Progress in traditional Chinese medicine treatment and research of uric acid nephropathy. Chin Med Modern Distance Edu Chin 16(09):158-160

10. Jing C (2019) Preliminary study on the construction of JNSFF quality control method based on spectrum-efficacy relationship. Chengdu Univ Tradi Chin Med. https://kns.cnki.net/kcms/ detail/detail.aspx?FileName $=1019632640$. nh\&DbName $=$ CMFD2 020

11. Fang W, Jing C, Yang L, Jing B, Haibo X, Jinlin G (2020) Study on pharmacodynamics and acute toxicity of antigout with antiuric acid compound. Nat Prod Res Dev 32(04):659-671

12. Yan L, Ji Z, Hang J, Yuanzhong W (2016) Application of chemical fingerprint in studies of edible and medicinal mushrooms. Food Sci 37(01):222-229

13. Fuyuan H, Kevin D, Huan Z, Yun Q, Feng C, Honghao Z (2011) Study on differences between pharmacokintics and chromatopharmacodynamics for Chinese materia medica formulae. China J Chin Mater Med 36(02):136-138

14. Jiaojiao L, Meng Y, Yuan G, Tao Z, Hongmei J, Hongwu Z, Liyan M, Zhongmei Z (2019) Anti-inflammatory active ingredient of Chinese herb Polygoni Cuspidati Rhizoma et radix based on spectrum-effect relationship. Chin J Exp Med Formul 25(12):208-213

15. Ming $Y(2018)$ Study on the spectral-effect relationship of antioxidant activity of Kudingcha. Guizhou normal University. https://kns.cnki.net/kcms/detail/detail.aspx?FileName $=10188$ 36738.nh\&DbName $=$ CMFD2019

16. Li L (2018) Study on the spectrum-effect relationship of nutmeg volatile oil in relieving chronic pain. Hubei Univ Tradit Chin Med.
https://kns.cnki.net/kcms/detail/detail.aspx?FileName=10181 66525.nh\&DbName $=$ CMFD2018

17. Pacher P, Nivorozhkin A, Szabó C (2006) Therapeutic effects of xanthine oxidase inhibitors: renaissance half a century after the discovery of allopurinol. Pharmacol Rev 58(1):87-114. https:// doi.org/10.1124/pr.58.1.6

18. Cheng Y, Li L, Zhou P, Zhang Y, Liu H (2019) Multi-objective optimization design and test of compound diatomite and basalt Fiber asphalt mixture. Materials (Basel) 12(9):1461. https://doi. org/10.3390/ma12091461

19. Sun X, Hu Z, Li M et al (2019) Optimization of pollutant reduction system for controlling agricultural non-point-source pollution based on grey relational analysis combined with analytic hierarchy process. J Environ Manag 243:370-380. https://doi.org/ 10.1016/j.jenvman.2019.04.089

20. Huang X, Zhang T, Yi G et al (2019) Dynamic changes of NDVI in the growing season of the Tibetan Plateau during the past 17 years and its response to climate change. Int J Environ Res Public Health 16(18):3452. https://doi.org/10.3390/ijerph16183452

21. Yang CT, Ghosh D, Beck JH, Jamie K, Kristi A, Kevin JM, Christina $G$ (2012) Screening for 250 pesticides in orange oil and ginseng extract by LC-MS/MS using TraceFinder software. Environ Chem 31(004):565-566

22. Caisheng W, Song W, Jinlan Z (2010) Separation and characterization of 4 related impurities in Puerarin bulk drug by HPLC-UVFTICRMS/MS ${ }^{n}$. J Chin Mass Spectr Soc 31(03):143-215

23. Zhihua $X$, Yuchang $M$, Xinping $L$, Bing $Z$, Mengdi $Z$, Shengmin $W$, Xin Y, Tianfeng Y, Jingwen J, Ran B (2017) Research progress of puerarin and its derivatives on anti-inflammatory and anti-gout activities. Chin J Chin Mate Med 42(19):3703-3708

24. Hongchang Z, Ying Z, Guangfu L, Enpeng W, Xin C (2016) The puerarin impact on the expression of $A B C G 2$ in human renal proximal tubule epithelial cells. Shanghai J Trad Chin Med 50(3):74-77

25. Yong W (2010) Effects of Myricetin and puerarin on hyperuricemia and its significance. Inner Mongolia J Tradit Chin Med 29(19):9-10

26. Liu Y, Che G, Di Z, Sun W, Tian J, Ren M (2020) Calycosin-7-O- $\beta$ D-glucoside attenuates myocardial ischemia-reperfusion injury by activating JAK2/STAT3 signaling pathway via the regulation of IL-10 secretion in mice. Mol Cell Biochem 463(1-2):175-187. https://doi.org/10.1007/s11010-019-03639-z

27. Yan X, Yu A, Zheng H, Wang S, He Y, Wang L (2019) Calycosin7-O- $\beta$-D-glucoside attenuates OGD/R-induced damage by preventing oxidative stress and neuronal apoptosis via the SIRT1/FOXO1/PGC-1a Pathway in HT22 Cells. Neural Plast 2019:8798069. https://doi.org/10.1155/2019/8798069

28. Fu S, Gu Y, Jiang JQ et al (2014) Calycosin-7-O- $\beta$-D-glucoside regulates nitric oxide/caveolin-1/matrix metalloproteinases pathway and protects blood-brain barrier integrity in experimental cerebral ischemia-reperfusion injury. J Ethnopharmacol 155(1):692-701. https://doi.org/10.1016/j.jep.2014.06.015

Publisher's Note Springer Nature remains neutral with regard to jurisdictional claims in published maps and institutional affiliations. 